\title{
OPTIMIZATION OF COOLING PIPE SYSTEM OF PLASTIC MOLDING
}

\author{
T. Matsumori, ${ }^{1}$ K. Yamazaki, ${ }^{2}$ and Y. Matsui ${ }^{2}$ \\ ${ }^{1}$ Graduated School of Natural Science \& Technology, Kanazawa University, Kakuma-machi, \\ Kanazawa, 920-1192, JAPAN, tadayosi@stu.kanazawa-u.ac.jp ${ }^{2}$ Kanazawa University, Kakuma- \\ machi, Kanazawa, 920-1192, JAPAN
}

\begin{abstract}
In a plastic injection molding, design of cooling pipe system is one of the important problems to reduce internal residual stresses of molded products. If plastic materials in the injection molding die are cooled down uniformly and slowly, generation of the residual stresses can be reduced.

In this paper, a new method to design a cooling pipe system in the plastic injection molding die taking account of coolant flow in the pipe are presented. To consider the effect of the coolant flow, two kinds of models assumed plastic injection die are prepared. And shape optimization techniques are applied to design the cooling pipe system in the models. To evaluate the optimality, two kinds of evaluation functions, one is to obtain uniform temperature distribution and the other is to control cooling rate, are defined.
\end{abstract}

keywords: Fluid Dynamics, Heat Transfer, Coupled Problem, Finite Element Method, Design Optimization

\section{Introductions}

Plastic injection molding is used widely to mold complex shapes of industrial products for mass production. In the plastic injection molding, one of the serious problems is the generation of residual strain and stress caused by non-uniform solidification of plastic materials. Cooling velocity of the injected plastic is slower than that of metal because of low heat conductivity of plastics. Therefore, differences of the cooling velocity part by part due to partial temperature gradient is appeared. These differences produce different molding shrinkage and internal residual stresses, which cause warps and cracks in the plastic molded products in some years after the molding. If the plastic materials in the solidification process are controlled well and cooled down uniformly, it is expected that the generation of the residual stress can be reduced much.

Many researchers have tried to optimize the cooling pipe system to reduce the generation of residual stresses by numerical simulation. The automatic

Please use the following format when citing this chapter:

Author(s) [insert Last name, First-name initial(s)], 2006, in IFIP International Federation for Information Processing, Volume 199, System Modeling and Optimization, eds. Ceragioli F., Dontchev A., Furuta H., Marti K., Pandolfi L., (Boston: Springer), pp. [insert page numbers]. 
design system of cooling pipes used straight line cooling pipe has been given $[1,2]$. Koresawa et al. suggested the automatic layout design system adopting complex cooling channels [3]. Recently, the plastic injection die with complex cooling channels can be produced by stereolithography system of metal powder. Therefore the complex layout will be adapted to plastic injection die.

However, in these researches, some factors such as a coolant flow in the cooling pipe have not been taken into consideration. The coolant flow has not been considered when a cooling pipe system is designed and optimized.

In this paper, we discuss about an optimization method of the cooling pipe system by taking coolant flow into account. For this purpose, two kinds of numerical models have been prepared; one is to confirm the validity of the evaluation function of objectives, the other is to consider the effect of the coolant flow in the process of optimization. These models are analyzed by the finite element code, which implements the heat transfer analysis and the fluid analysis. And the temperature distribution and its variation as well as the coolant velocity during the cooling process calculated by the analyses are adopted to optimize the cooling pipe system. Then, to decide the position of the cooling pipe, the basis vector method [4], which is one of the ways to treat a shape change and to control mesh adaptation, is adopted. In the basis vector method, the design variables are set by using the orthogonal array of the design of experiment (DOE). From the numerical analysis results the response surfaces are constructed. Then the optimum shape of the cooling pipe system is obtained by the mathematical programming method.

\section{How to optimize a cooling pipe system}

\subsection{Optimization procedure}

In this research, the shape and the position of the cooling pipe system are taken as the design variables. The sampling points are assigned by the orthogonal array in DOE, and the numerical simulations have been implemented. Then the response surface for the evaluation fanctions are constructed and optimized. Figure 1 shows flow chart of the design processes.

\subsection{Numerical model}

The objective of this paper is to establish an optimization methodology for the cooling pipe system considering the coolant flow effect. To confirm the effect of suggested methodology, a numerical model shown in Figure 2, which is two dimensional model, with two kinds of different conditions, which are called "Case 1" and "Case 2", is prepared. The Case 1 does not consider the coolant flow effects. Therefore only the heat transfer analysis will be done. On the other hand, the Case 2 takes account of the coolant flow, which requires 
analyzing both the fluid flow and the heat transfer. And we make the numerical model to compare the optimization results between Case 1 and Case 2. If the optimum shape of Case 2 is different from the shape of Case 1, we can confirm the effect of coolant flow.

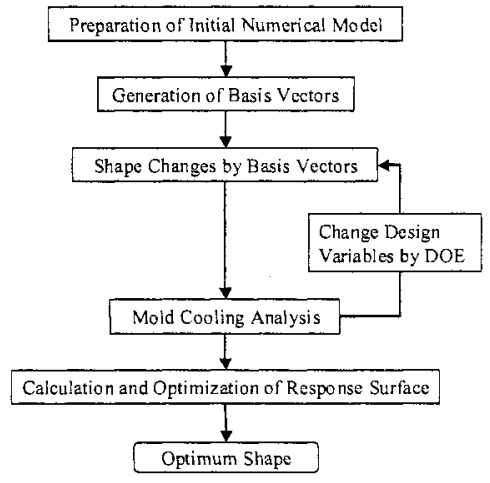

Figure 1. Flow chart to optimize cooling pipe system

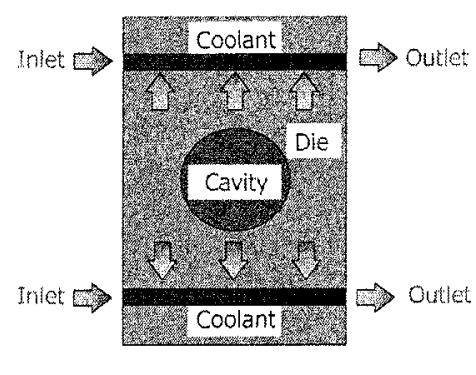

Figure 2. Numerical model

In the numerical analysis, we consider only half of the numerical model because the conditions of the model are given symmetric with respect to the $\mathrm{x}$-axis.

\subsection{Basis vector method}

When an optimization method is applied to the shape design problem, the basis vector method is adopted to decide the shape and position of cooling pipe, and to reduce the number of design variables. The basis vector method can prevent the mesh warping due to the boundary shape changes during optimization. In the basis vector method, an original shape vector and basis vectors need to be prepared. In general, a new coordinate $\mathbf{G}$ is generated from the following equation,

$$
\mathbf{G}=\mathbf{G}_{0}+\sum_{i=1}^{n} \alpha_{i}\left(\mathbf{V}_{i}-\mathbf{G}_{0}\right)
$$

where $\mathbf{G}_{0}$ is an original shape vector, $\mathbf{V}_{i}$ is a basis vector, $n$ is the number of basis vectors and $\alpha_{i}$ is a design variable, respectively.

In this study, the new shape vector is calculated from the following equation,

$$
\mathbf{G}=\mathbf{G}_{0}+\sum_{i=1}^{n} \alpha_{i} \sum_{j=1}^{n o n} \mathbf{V}_{j}
$$


where non is the number of node in a numerical model and $\mathbf{V}_{i}$ corresponds to $\left(\mathbf{V}_{i}-\mathbf{G}_{0}\right)$.

Four kinds of basis vectors that include four design variables are prepared. Figure 3 illustrates the shape changes of the numerical model by using the basis vectors.

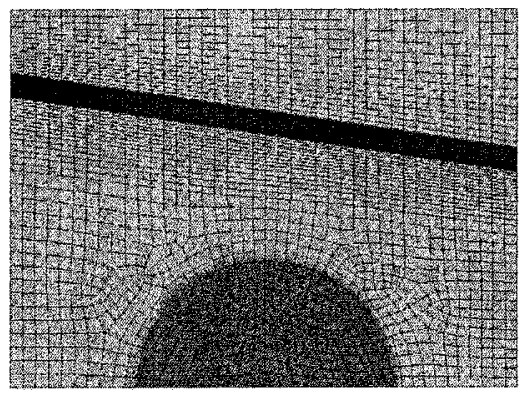

(a) Inclined straight pipe

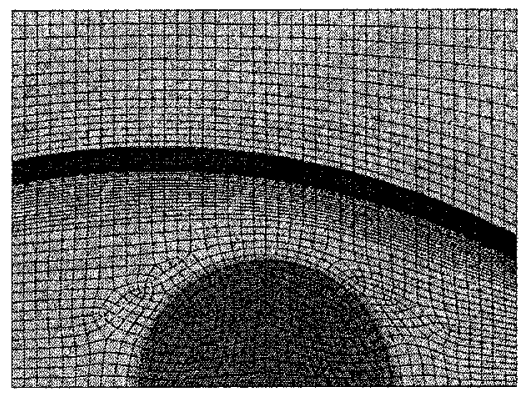

(b) Curved pipe

Figure 3. Examples of shape change using basis vectors

\subsection{Response surface approach $[5,6]$}

In the optimization problem using numerical analyses, when much calculation time is required for one analysis, the design space may be approximated to optimize effectively. Some approximate optimization techniques have been developed, such as the response surface method (RSM), Kriging model, the radial basis function (RBF) and so on. In this study, we have applied the RSM to predict the numerical results without a lot of analyses in optimization process.

When the shape of numerical model is changed by using basis vector method, a few numbers of design variables have to be decided. In this study, the combination of these design variables is determined by an orthogonal array of the design of experiment (DOE) to be calculated efficiently under required reliability. And an orthogonal polynomial calculated by a regression model based on the array is regarded as the response surface.

\subsection{Evaluation function}

In a plastic injection molding, when the temperature distribution of melted plastic in a cavity is uniform and the cooling rate is slow, the generation of residual stresses is suppressed. Therefore, we introduce two kinds of quantities to evaluate these conditions. One is an average heat quantity $Q_{\text {ave }}$ defined by Eq.(3) to obtain uniform temperature distribution along the cavity surface. The 
other is a deviation of heat quantity $Q_{\operatorname{dev}}$ defined by Eq.(4) to measure the cooling rate during $t_{\text {end }}$ in the molding die.

$$
\begin{gathered}
Q_{\text {ave }}=\int_{0}^{t_{\text {end }}} q_{\text {ave }} d t=-k A \int_{0}^{t_{\text {end }}} \frac{\sum_{i=1}^{n} T_{i}}{n} d t \\
Q_{\mathrm{dev}}=\int_{0}^{t_{\text {end }}} q_{\text {dev }} d t=-k A \int_{0}^{t_{\text {end }}} \sqrt{\frac{\sum_{i=1}^{n}\left(T_{i}-T_{\text {ave }}\right)^{2}}{n-1} d t}
\end{gathered}
$$

where $q_{\mathrm{ave}}$ and $q_{\mathrm{dev}}$ are heat flows of the cavity surface nodes at unit time, $t_{\mathrm{end}}$ is calculation time, $k$ is thermal conductivity, $A$ is an area of the cavity surface, $T_{i}$ is a temperature of cavity surface node, $T_{\text {ave }}$ is an average temperature of the surface and $n$ is number of the nodes, respectively.

After normalizing these two functions, an objective function summed up these functions with weighted coefficients is introduced to transform this multiobjective optimization problem into a single one.

$$
f=w_{1} Q_{\text {ave }}+w_{2} Q_{\text {dev }} \quad\left(w_{1} \geq 0, w_{2} \geq 0, w_{1}+w_{2}=1\right)
$$

where $w_{1}$ and $w_{2}$ are weighted coefficients.

\section{Shape optimization problem of cooling pipe system in plastic injection molding}

\subsection{Optimum shape of cooling pipe system}

A simple design model of cooling pipe system (Figure 2) is considered to confirm the coolant flow effects between the different conditions, the Case 1 and the Case 2, as mentioned above. The same initial conditions are assumed for the both cases. As the boundary conditions, a constant cool temperature is given for all area of cooling pipe in the Case 1. On the other hand, in the Case 2 a constant velocity and temperature at inlet, a constant pressure at outlet and no slip condition at cooling pipe wall are assumed to consider the coolant flow in cooling pipe.

The analytical models are made from the combination of the basis vectors based on the orthogonal array $L_{27}\left(3^{4}\right)$. Using 27 kinds of numerical results analyzed these models by the FEM, the response surface is constructed.

The response surfaces in the design space are illustrated in Figs.4. These graphs show the relationship among the average heat quantity, the deviation of heat quantity and the transformed single objective function. By changing the coefficient values of $w_{1}$ and $w_{2}$ in Eq.(5), a Parato front drawn by red symbol is obtained.

The optimum shapes of cooling pipe systems shown in Figs.5 and 6 are obtained by using the response surfaces (Eq.(5)) and the mathematical programming method under the condition of $w_{1}=0.5, w_{2}=0.5$. The optimum 

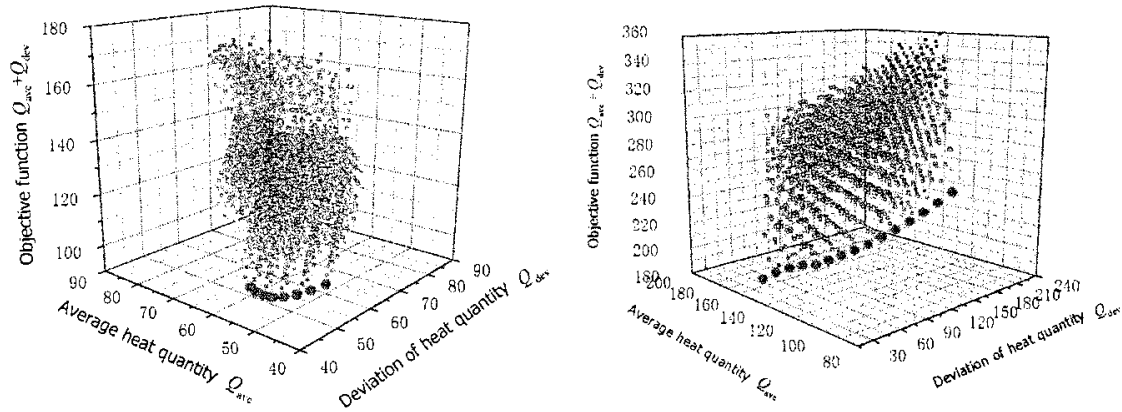

Figure 4. Response surface of Case 1 (left), Case 2 (right)

shape of cooling pipe in the Case 1 shows round and symmetric as shown in Figure 5 because of the symmetry of simulation model. On the other hand, the shape is asymmetry and that the cooling pipe approaches the cavity toward outlet and goes away from the cavity toward inlet in the Case 2 (Figure 6).

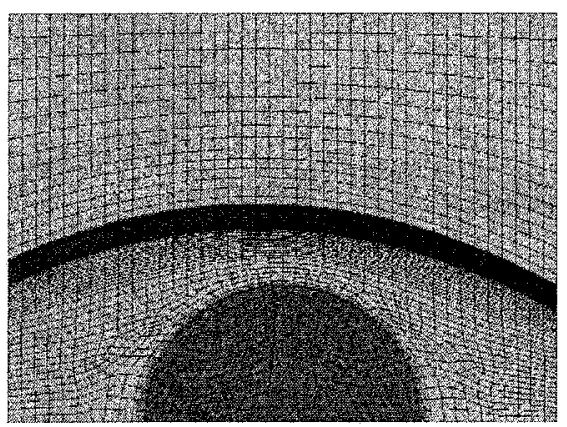

Figure 5. Optimum shape of Case1

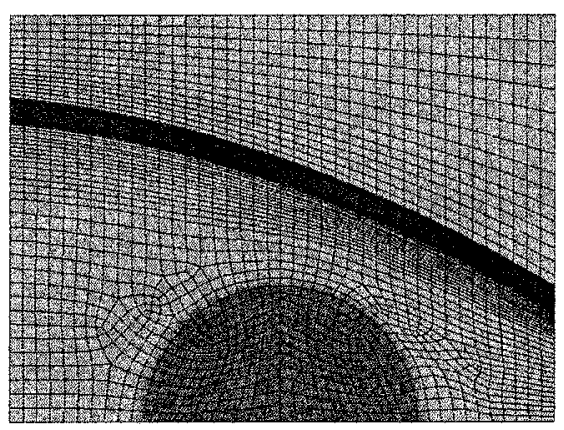

Figure 6. Optimum shape of Case2

\subsection{Discussions}

The optimum shape in Case 1 can be expected to reduce the differences of the temperatures distribution and to cool down fast on the cavity surface. Compared with the optimized model in Case 1, we can find clearly the difference of the inlet and outlet coordinate of the cooling pipe. When the coolant flow is considered, the coolant temperature of inlet side is higher than that of outlet side because of the absorption of heat. This will cause the nonuniform temperature distribution 
in the die. From this reason, the asymmetry optimum shape in Case 2, has been obtained in order to make a temperature distribution uniformly.

In Figs.5 and 6, the Parato fronts are obtained to optimize the objective function with the different values of the weights. The pipe shapes of these solutions in Figure 6 are illustrates in Figure 7. The trade off relationship between the average heat quantity $Q_{\text {ave }}$ and the deviation of heat quantity $Q_{\mathrm{dev}}$ is observed. From the shapes of Parato solution, we can recognize that it is necessary to make the position of inlet go away from the cavity for uniform temperature distribution.

When the optimum results shown in Figure 6 are focused, the result of deviation of heat quantity is the minimum at the optimized results as shown Figure 7. In Figure 7, the result of average heat quantity is not the minimum of objective function, because the function value depends on the normalizing standard. However, changing the weighted coefficient, Parato front is obtained. Therefore, we consider the objective function and evaluation functions show a natural phenomenon in plastic injection molding.

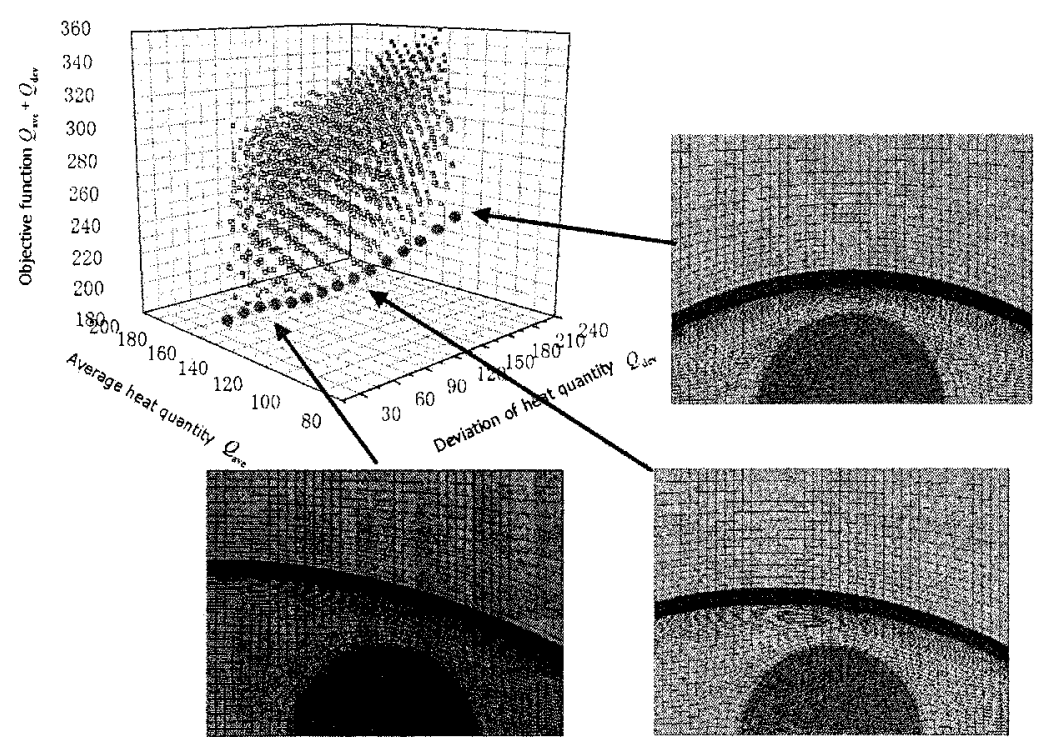

Figure 7. Shapes of parato solutions in Case2

\section{Conclusions}

A new approach to optimize the cooling pipe system in the plastic injection molding taking account of the coolant flow has been developed. To confirm the 
coolant flow effect, we prepared a simple cooling pipe system model, and optimized the model with two different conditions. In the optimization processes, we defined the two kinds of quantities to evaluate the cooling process in the cavity of plastic injection die, and adapted the basis vector method to control the shape of cooling pipe system and the response surface method to optimize effectively. As a result, we obtained the different optimum shapes that appear the coolant flow effect. And the response surfaces using the optimizations express the trade off relation ship between the cooling rate and the uniformity of the temperature distribution in the die. However, the optimum shape is lean to keep the uniformity compared with the cooling rate when two evaluation functions are treated at the same rate. However, we can obtain the parato front to change the weight of two functions.

\section{References}

[1] C.L.Li, Automatic layout design of plastic injection mould cooling system, Computer Aided Design , 33:1073-1090, 2001.

[2] C.L. Li, C.G. Li, A.C.K. Mok, A feature-based approach to injection mould cooling system design, Computer Aided Design 37:645-662, 2005.

[3] H. KORESAWA, Y. TOCHIKA, H. SUZUKI, Automatic design for cooling channels in injection molding under steady-state heat conduction analysis, Transactions of the Japan Society of Mechanical Engineers Series C, 2087-2093, 1999.

[4] Genesis user's manual, VR\&D Inc., 2000.

[5] T. KASHIWAMURA et al., Optimization of nonlinear problem by experimental design, Asakura shoten, 1998, (in Japanese).

[6] R. H. Myers, D. C. Montgomery, Response surface methodology, Wiley, 1995. 